ORIGINAL PAPER

\title{
Aldehyde Dehydrogenase- 1 positivity is associated WITH ER NEGATIVITY IN PATIENTS WITH INVASIVE DUCTAL CARCINOMA OF THE BREAST
}

\author{
Yazgi Köy ${ }^{1}$, Fikret Dirilenoglu$^{2}$, Ümit S. Tetikkurt $^{3}$, Ali MuhammedoĞlu ${ }^{3}$, Atilla Çelik $^{4}$
}

${ }^{1}$ Department of Pathology, Batman State Hospital, Batman, Turkey

${ }^{2}$ Department of Pathology, Near East University, Faculty of Medicine, Cyprus

${ }^{3}$ Department of Pathology, University of Health Sciences, Bağcılar Traning and Research Hospital, Istanbul, Turkey

${ }^{4}$ Department of General Surgery, University of Health Sciences, Bağcllar Training and Research Hospital, Istanbul, Turkey

Cancer stem cells (CSCs) are self-renewable and can be differentiated into different cell types. They play an important role in oncogenic signaling pathways, tumor cell heterogeneity, metastasis, and therapeutic resistance. Aldehyde dehydrogenase 1 (ALDH1) was identified as a specific marker for breast CSCs. The study included a total of 105 patients with a diagnosis of invasive ductal carcinoma (IDC) who underwent mastectomy and with sufficient pathology material for histopathological examination. Patient demographics, tumor location, tumor diameter, the presence of lymphovascular and perineural invasion and lymph node metastasis, surgical margin status, and immunohistochemistry (IHC) staining results were obtained from patients' records. The tumors were classified into IHC-based molecular subtypes according to the St. Gallen Consensus Conference in 2013. A four-tiered scoring system was used based on ALDH1 staining percentage in tumor cells. The tumor was determined as positive if the score was 2 or higher. Clinical, histopathological findings, and ALDH1 staining results were correlated. Twenty-five cases (23.8\%) were ALDH1 positive. The ALDH1 positive group compared to the negative group was found to be associated with ER negativity $(p=0.044)$, but there was no correlation with other clinical and histopathological findings. ALDH1-positive IDCs may be less sensitive to hormonal therapy and associated with aggressive behavior.

Key words: aldehyde dehydrogenase 1, stem cell, breast, invasive ductal carcinoma, pathology.

\section{Introduction}

Cancer stem cells (CSCs), which are self-renewable and can be differentiated into different cell types, play an important role in oncogenic signaling pathways, tumor cell heterogeneity, metastasis, and therapeutic resistance $[1,2,3]$. The presence of CSCs has been shown in various types of malignancies, such as colon, brain, lung, and breast tumors $[2,4,5,6,7]$. In 2003, the breast CSCs were first isolated by their
$\mathrm{CD} 44^{+} \mathrm{CD} 24^{- \text {llow }}$ surface marker expression [6]. In 2007, expression of a single marker, aldehyde dehydrogenase 1 (ALDH1), was shown to be specific for normal and malignant human breast stem cells [7].

Aldehyde dehydrogenase functions to catalyze the oxidation of aldehydes to their relevant carboxylic acids [8]. To date, 19 different ALDH functional genes and multiple splice variants have been described [9]. Aldehyde dehydrogenase 1 is one of the isoforms playing an important role in the retinoic 
acid pathway through the catalysis of retinaldehyde to retinoic acid [10]. Isolated cancer cells with relatively high ALDH1 activity were shown to have in vitro features of CSCs, including capabilities of proliferation, self-renewal, and differentiation, and resistance to chemotherapy $[7,11,12]$.

In this study, we aimed to determine the clinical and pathological value of immunohistochemical (IHC) staining of ALDH1 in tumor cells as well as peritumoral and intratumoral stromal cells in a series of invasive ductal carcinomas (IDCs).

\section{Material and methods}

This study was approved by the non-interventional clinical research ethical board (No: 2016/462).

\section{Patients and clinical information}

We retrospectively identified the patients diagnosed with IDC in the pathology laboratory between 2010 and 2016. We included all the patients who underwent mastectomy and with sufficient pathology material for histopathological examination. Clinical data were retrieved from the electronic medical records.

\section{Review of histopathological and immunohistochemical findings}

Two pathologists (YK and ÜT) reviewed all hematoxylin-eosin slides received from the pathology archive and determined the Nottingham histological grade of each tumor [13]. Patient demographics, tumor location, tumor diameter, the presence of lymphovascular and perineural invasion, surgical margin status, the presence of lymph node metastasis, and IHC staining results were obtained from patients' records and pathology reports. The status of estrogen receptor (ER), progesterone receptor (PR), and human epidermal growth factor receptor 2 (HER2) was determined according to the American Society of Clinical Oncology/College of American Pathologists guidelines $[14,15]$. The Ki-67 proliferation index was regarded as high if there was more than $14 \%$ staining in tumor cells [16]. All cases with available IHC results were divided into molecular subtypes according to the International Breast Cancer Conference in St Gallen in 2013 [16]. Microscopic examination was performed using an Olympus brand MDOB3 model 8H16329 serial microscope.

\section{Aldehyde dehydrogenase 1 staining and evaluation method}

One representative block from each case was selected for staining with ALDH1 (polyclonal antibody, IgG isotype, dilution 1 : 150; Gene'Tex, Irvine,
CA, USA). Sections with a thickness of four microns were taken to positive-charged slides. The sections were left for 60 minutes at a temperature of $60^{\circ} \mathrm{C}$. Then, the sections were kept at room temperature for 10 minutes and automatically stained with the XT $\mathrm{DAB} V 3$ protocol based on multimer technology in the Ventana BenchMark XT model. After the staining process, the sections were washed in soapy water for 5-10 minutes. After that, the slides were dried, placed in $96 \%$ alcohol and xylene, then they were closed in the Sakura Tissue Single Film model automatic film closure device.

We evaluated and scored ALDH1 immunostaining in the tumor cells as follows: score 0 , no staining in the tumor cells; score 1, staining in less than $10 \%$ of the tumor cells; score 2, staining in more than $10 \%$ but less than $50 \%$ of the tumor cells; score 3 , staining in more than $50 \%$ of the tumor cells. In the following analysis, we determined the tumor as ALDH1 positive if the tumor had a score of 2 or higher as previously described [17]. We also examined the stromal cell staining using a $10 \%$ cutoff value for positivity [18]. Adult liver tissue was used as a positive control.

\section{Statistical analysis}

Histopathological and clinical findings and IHC results were tabulated. All statistical analyses were carried out using the IBM SPSS Statistics 22.0 package program (IBM Corp., Armonk, New York, USA). The frequencies of clinical and histopathologic variables were presented by using cross-tabulations. A two-sided Fisher's $\chi^{2}$ exact test for $r x c$ tables was applied to compare the differences between groups for categorical variables. The normal distribution of variables was examined visually (histogram and probability plots) and with analytical methods (Shapiro-Wilk tests). A p value of less than 0.05 was considered significant.

\section{Results}

\section{Patients and clinical information}

There were a total of 105 patients including two males with a diagnosis of IDC. All cases had sufficient pathology material and clinicopathologic data.

The median age was 50 (range 24-85). Fifty-two $(49.5 \%)$ of the tumors were located in the right breast, 48 (45.7) were in the left, and five (4.8\%) involved both. The cases predominantly underwent modified radical mastectomy ( $\mathrm{n}=70,66.7 \%$, including two cases with bilateral involvement), 24 (22.9) cases had a partial mastectomy, and eight cases (7.6\%) had a simple mastectomy. Three out of five cases with 

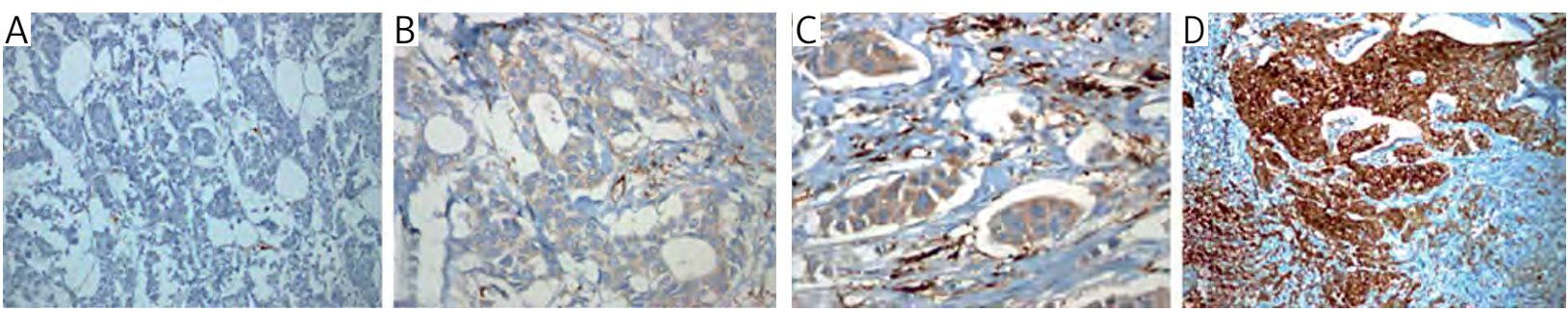

Fig. 1. ALDH1 staining in invasive ductal carcinomas. A) Score 0, negative for ALDH1. B) Score 1, negative for ALDH1. C) Score 2, positive for ALDH1. D) Score 3, positive for ALDH1
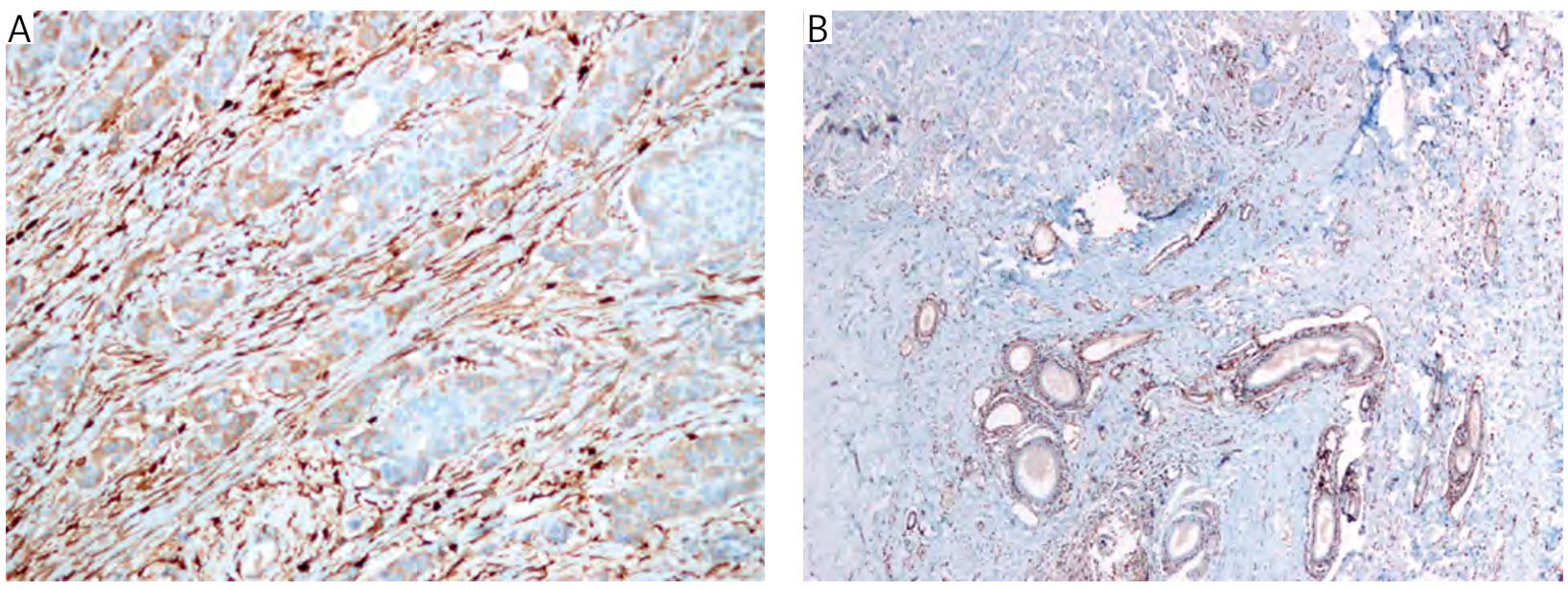

Fig. 2. ALDH1 staining in stromal cells in invasive ductal carcinomas. A) ALDH1 staining in intratumoral stromal cells. B) ALDH1 staining particularly in peritumoral and periductal stromal cells

bilateral breast carcinoma had simple mastectomy and modified radical mastectomy during the same operation.

\section{Review of histopathological and immunohistochemical findings}

The median diameter of the tumors was $25 \mathrm{~mm}$ (range $5-70 \mathrm{~mm}$ ). Eighty-four tumors $(80 \%)$ had a single focus, $16(15.2 \%)$ were multifocal, and the remainder were bilateral. According to the histological grading system, 78 cases $(74.3 \%)$ were grade 3, 26 cases $(24.8 \%)$ were grade 2 , and one case $(1 \%)$ was grade 1 . Lymphatic invasion was present in 66 cases $(62.9 \%)$ and vascular invasion was seen in 25 cases $(23.8 \%)$. Lymph node metastasis was detected in 70 cases $(66.7 \%)$. Ninety cases $(85.7 \%)$ were ER positive, $80(76.2 \%)$ were PR positive, 28 out of 81 cases $(34.6 \%)$ were HER2 positive, and Ki-67 was high in $73(69.5 \%)$ cases. Classification of the tumors based on the IHCbased molecular subtypes $(\mathrm{n}=100)$ revealed that 62 cases $(62 \%)$ were in luminal B, 24 cases $(24 \%)$ were in luminal A, 12 cases (12\%) were in HER2, and 2 cases $(2 \%)$ were in triple-negative breast carcinoma (TNBC) subtype.

\section{Aldehyde dehydrogenase 1 staining in tumor and stromal cells}

Aldehyde dehydrogenase 1 staining in IDCs is displayed in Fig. 1. Aldehyde dehydrogenase 1 was recorded as score 0 in 21 cases (20\%), score 1 in 59 cases $(56.2 \%)$, score 2 in 18 cases $(17.1 \%)$, and score 3 in 7 cases $(6.7 \%)$. The tumor cells in 25 cases $(23.8 \%)$ were ALDH1 positive. All cases showed positivity in peritumoral stromal areas. All cases but one showed positivity in intratumoral stromal areas (Fig. 2). In addition, the concentration of ALDH1-positive stromal cells was more prominent in the peritumoral areas compared to the stromal cells surrounding benign ducts within tumor cells.

\section{Correlation of aldehyde dehydrogenase 1 staining in tumor cells with clinical and histopathological features}

The ALDH1 positive group compared to the negative group was found to be associated with ER negativity $(p=0.044)$. Aldehyde dehydrogenase 1 staining and IHC-based molecular subtypes were found to have a significant association $(p=0.025)$. A pairwise z-test post hoc analysis with Bonferroni correction revealed that only for TNBC was there a significant 
Table I. Correlation of ALDH1 staining in the tumor cells with the clinicopathological parameters in invasive ductal carcinomas

\begin{tabular}{|c|c|c|c|c|c|}
\hline \multirow{3}{*}{$\begin{array}{l}\text { Clinical and Pathological } \\
\text { Parameters }\end{array}$} & \multicolumn{4}{|c|}{ ALDH1 IN TUMOR } & \multirow[t]{3}{*}{ P VALUE } \\
\hline & \multicolumn{2}{|c|}{ Negative } & \multicolumn{2}{|c|}{ Positive } & \\
\hline & $\#$ & $\%$ & $\#$ & $\%$ & \\
\hline \multicolumn{6}{|l|}{ Age group (years) } \\
\hline$\leq 40$ & 17 & $(77.3)$ & 5 & $(22.7)$ & \\
\hline $40-55$ & 31 & $(79.5)$ & 8 & $(20.5)$ & 0.766 \\
\hline$\geq 55$ & 32 & $(72.7)$ & 12 & $(27.3)$ & \\
\hline \multicolumn{6}{|l|}{ Diagnosis } \\
\hline IDC & 74 & $(75.5)$ & 24 & $(24.5)$ & \multirow[t]{2}{*}{0.683} \\
\hline IDC with micropapillary component & 6 & $(85.7)$ & 1 & $(14.3)$ & \\
\hline \multicolumn{6}{|l|}{ Laterality } \\
\hline Right & 40 & $(76.9)$ & 12 & $(23.1)$ & \\
\hline Left & 36 & $(75)$ & 12 & $(25)$ & 1.000 \\
\hline Bilateral & 4 & $(80)$ & 1 & $(20)$ & \\
\hline \multicolumn{6}{|l|}{ Operation } \\
\hline $\mathrm{PM}$ & 20 & $(83.3)$ & 4 & $(16.7)$ & \multirow[t]{4}{*}{0.568} \\
\hline SM & 6 & $(75)$ & 2 & $(25)$ & \\
\hline MRM & 51 & $(72.9)$ & 19 & $(27.1)$ & \\
\hline $\mathrm{SM}+\mathrm{MRM}$ & 3 & $(100)$ & 0 & $(0)$ & \\
\hline \multicolumn{6}{|l|}{ Number of foci } \\
\hline Single focus & 67 & $(79.8)$ & 17 & $(20.2)$ & \\
\hline Multifocal & 9 & $(56.3)$ & 7 & $(43.8)$ & 0.113 \\
\hline Bilateral & 4 & $(80)$ & 1 & $(20)$ & \\
\hline \multicolumn{6}{|l|}{ Diameter } \\
\hline$\leq 2 \mathrm{~cm}$ & 23 & $(85.2)$ & 4 & $(14.8)$ & \\
\hline $2-5 \mathrm{~cm}$ & 53 & $(73.6)$ & 19 & $(26.4)$ & 0.526 \\
\hline$\geq 5 \mathrm{~cm}$ & 4 & $(66.7)$ & 2 & $(33.3)$ & \\
\hline \multicolumn{6}{|l|}{ Histological grade } \\
\hline Grade I-II & 20 & $(74.1)$ & 7 & $(25.9)$ & \multirow[t]{2}{*}{0.796} \\
\hline Grade III & 60 & $(76.9)$ & 18 & $(23.1)$ & \\
\hline \multicolumn{6}{|l|}{ Lymph node metastasis } \\
\hline Absent & 26 & $(74.3)$ & 9 & $(25.7)$ & \multirow[t]{2}{*}{0.810} \\
\hline Present & 54 & $(77.1)$ & 16 & $(22.9)$ & \\
\hline \multicolumn{6}{|l|}{ Lymphatic invasion } \\
\hline Absent & 29 & $(74.4)$ & 10 & $(25.6)$ & \multirow[t]{2}{*}{0.814} \\
\hline Present & 51 & $(77.3)$ & 15 & $(22.7)$ & \\
\hline \multicolumn{6}{|l|}{ Vascular invasion } \\
\hline Absent & 63 & $(78.8)$ & 17 & $(21.3)$ & \multirow[t]{2}{*}{0.290} \\
\hline Present & 17 & (68) & 8 & (32) & \\
\hline \multicolumn{6}{|l|}{ ER } \\
\hline Negative & 8 & $(53.3)$ & 7 & $(46.7)$ & \multirow[t]{2}{*}{0.044} \\
\hline Positive & 72 & $(80)$ & 18 & $(20)$ & \\
\hline
\end{tabular}


Table I. Cont.

\begin{tabular}{|c|c|c|c|c|c|}
\hline \multirow{3}{*}{$\begin{array}{l}\text { Clinical and Pathological } \\
\text { Parameters }\end{array}$} & \multicolumn{4}{|c|}{ ALDH1 IN TUMOR } & \multirow[t]{3}{*}{ P VALUE } \\
\hline & \multicolumn{2}{|c|}{ Negative } & \multicolumn{2}{|c|}{ Positive } & \\
\hline & $\#$ & $\%$ & $\#$ & $\%$ & \\
\hline \multicolumn{6}{|l|}{ PR } \\
\hline Negative & 17 & $(68)$ & 8 & $(32)$ & \multirow[t]{2}{*}{0.290} \\
\hline Positive & 63 & $(78.8)$ & 17 & $(21.3)$ & \\
\hline \multicolumn{6}{|l|}{ HER2 } \\
\hline Negative & 40 & $(75.5)$ & 13 & $(24.5)$ & \multirow[t]{2}{*}{0.963} \\
\hline Positive & 21 & $(75)$ & 7 & $(25)$ & \\
\hline \multicolumn{6}{|l|}{$\mathrm{Ki}-67$} \\
\hline Low $(\leq 14)$ & 26 & $(81.3)$ & 6 & $(18.8)$ & \multirow[t]{2}{*}{0.467} \\
\hline $\operatorname{High}(>14)$ & 54 & $(74)$ & 19 & (26) & \\
\hline \multicolumn{6}{|l|}{ Molecular subtype } \\
\hline Luminal A & 20 & $(83.3)$ & 4 & $(16.7)$ & \multirow[t]{4}{*}{0.025} \\
\hline Luminal B & 49 & $(79)$ & 13 & $(21)$ & \\
\hline HER2 & 7 & $(58.3)$ & 5 & $(41.7)$ & \\
\hline TNBC & 0 & $(0)$ & 2 & $(100)$ & \\
\hline
\end{tabular}

difference between the ALDH1 positive and negative cases $(\mathrm{p}<0.05)$. There was no significant relationship between ALDH1 positivity and the other clinicopathologic parameters (Table I).

\section{Discussion}

Aldehyde dehydrogenase 1 was identified as a reliable stem cell marker of the normal human breast and breast carcinomas [7]. In this study, our purpose was to appraise the clinical and pathological value of ALDH1 staining in patients with a diagnosis of IDC, no special type. Our results showed that ALDH1-positive IDCs are associated with ER negativity. Hormone receptor negativity in invasive breast carcinomas is a well-known factor associated with treatment resistance and poor prognosis; hence, our results suggested that ALDH1-positive IDCs may represent a biologically aggressive phenotype $[4,19]$.

In breast carcinomas, ALDH1 positivity has ranged from $18 \%$ to $56 \%$ in different studies $[7,20$, $21,22,23]$. Likewise, $23.8 \%$ of IDCs in our series were positive for ALDH1. Various studies have aimed to determine the clinical and pathological differences between ALDH1-positive and negative breast carcinomas. In accordance with our results, some of these studies have shown that ALDH1 positivity is associated with ER negativity $[7,17,18,24,25]$. Some studies have found that ALDH1 positive breast carcinomas are also more likely to be PR negative and/or
HER2 positive, and they are associated with a high $\mathrm{Ki} 67$ proliferative index; but some others including our study have found no such correlation $[7,17,18$, 24, 25, 26, 27]. Several studies have demonstrated that ALDH1-positive invasive breast carcinomas are associated with TNBC and/or HER2(+) subtype $[17,18,26,27]$. In our series, ALDH1 positivity was observed more frequently in TNBCs as well; however, the number of TNBC patients was too small to determine an exact association. There are discrepancies in studies correlating ALDH1 positivity and histological grade, tumor size, and patient outcome $[6,7,17,18,24,25,26,27,28,29]$. Most studies have reported no value of finding of ALDH1-positive tumor cells in predicting lymph node metastasis $[7$, $17,18,24,26,27]$. In our study, we did not find an association with ALDH1 positivity and several significant prognostic factors, such as tumor diameter, histological grade, lymph node metastasis, lymphatic or vascular invasion.

Aldehyde dehydrogenase 1 staining in intratumoral stromal and peritumoral stromal cells in breast cancers has received special attention. One study reported high levels of ALDH1 staining in stromal cells in breast cancers that develop sporadically or in patients with BRCA1 mutations. Peritumoral stromal staining in the BRCA1-mutated group was significantly higher than the non-BRCA1-mutated group. According to this study, peritumoral stromal ALDH1 staining was associated with ER and PR negativity, basal-like phenotype, and high mitotic activity [30]. 
In our study, ALDH1 staining in the peritumoral stromal cells was observed in all but one case. Our findings are incompatible with the above study. Another observation of ours was that ALDH1-positive stromal cells were more concentrated in the peritumoral areas compared to the stromal cells surrounding benign ducts within tumor cells. In one study, ALDH1, which takes part in the retinoic acid synthesis pathway, was shown to have a role in normal epithelial cell proliferation and their ability to acquire progenitor/stem cell differentiation properties [31]. In another study, retinoic acid-producing ALDH1-positive dendritic cells in the intestines were shown to activate immune system cells. In addition, it has been found that the presence of ALDH1-positive stromal cells in tumors increases retinoic acid synthesis and secretion, thus increasing tumor cell differentiation and reducing the aggressiveness of the tumor [18]. These findings have suggested that activated immune system cells may play a role in preventing the development of malignancy in the areas where ALDH1-positive stromal cell density is high around the tumor, as seen in our cases.

There were a few limitations of our study. The number of patients included in this study was relatively small. This was particularly a drawback in the correlation analysis between ALDH1 staining and molecular subtypes. Survival analyses were not carried out in this study; hence we have limited results regarding the prognostic value of ALDH1.

In conclusion, our findings suggest that stem celllike features are more prominent in IDCs with ER negativity. Hence, ALDH1-positive IDCs may be more aggressive and less sensitive to hormonal therapy. However, further studies would be needed to reveal whether there is any prognostic significance of ALDH1. It is plausible that the peritumoral concentration of ALDH1-positive stromal cells may generate a reactive stromal response against the development of malignancy or may be protective.

\section{The authors declare no conflict of interest.}

\section{References}

1. Meacham CE, Morrison SJ. Tumour heterogeneity and cancer cell plasticity. Nature 2013; 501: 328-337.

2. Singh SK, Clarke ID, Terasaki M, et al. Identification of a cancer stem cell in human brain tumors. Cancer Res 2003; 63: 5821-5828.

3. Diehn M, Cho RW, Lobo NA, et al. Association of reactive oxygen species levels and radioresistance in cancer stem cells. Nature 2009; 458: 780-783.

4. Osborne CK, Yochmowitz MG, Knight WA, McGuire WL. The value of estrogen and progesterone receptors in the treatment of breast cancer. Cancer 1980; 46: 2884-2888.

5. Lapidot T, Sirard C, Vormoor J, et al. A cell initiating human acute myeloid leukaemia after transplantation into SCID mice. Nature 1994; 367: 645-648.
6. Al-Hajj M, Wicha MS, Benito-Hernandez A, et al. Prospective identification of tumorigenic breast cancer cells. Proc Natl Acad Sci 2003; 100: 3983-3988.

7. Ginestier C, Hur MH, Charafe-Jauffret E, et al. ALDH1 Is a marker of normal and malignant human mammary stem cells and a predictor of poor clinical outcome. Cell Stem Cell 2007; 1: 555-567.

8. Rodriguez-Torres M, Allan AL. Aldehyde dehydrogenase as a marker and functional mediator of metastasis in solid tumors. Clin Exp Metastasis 2016; 33: 97-113.

9. Vasiliou V, Nebert DW. Analysis and update of the human aldehyde dehydrogenase (ALDH) gene family. Hum Genomics 2005; 2: 138-143.

10. Chute JP, Muramoto GG, Whitesides J, et al. Inhibition of aldehyde dehydrogenase and retinoid signaling induces the expansion of human hematopoietic stem cells. Proc Natl Acad Sci USA 2006; 103: 11707-11712.

11. Jiang F, Qiu Q, Khanna A, et al. Aldehyde dehydrogenase 1 is a tumor stem cell-associated marker in lung cancer. Mol Cancer Res 2009; 7: 330-338.

12. Croker AK, Allan AL. Inhibition of aldehyde dehydrogenase (ALDH) activity reduces chemotherapy and radiation resistance of stem-like ALDH hiCD44 + human breast cancer cells. Breast Cancer Res Treat 2012; 133: 75-87.

13. Sugawara E, Nikaido H. Properties of AdeABC and AdeIJK efflux systems of Acinetobacter baumannii compared with those of the AcrAB-TolC system of Escherichia coli. Antimicrob Agents Chemother 2014; 58: 7250-7257.

14. Hammond MEH, Hayes DF, Dowsett M, et al. American Society of Clinical Oncology/College of American Pathologists guideline recommendations for immunohistochemical testing of estrogen and progesterone receptors in Breast Cancer. J Clin Oncol 2010; 28: 2784-2795.

15. Wolff AC, Hammond MEH, Hicks DG, et al. Recommendations for human epidermal growth factor receptor 2 testing in breast cancer : American Society of Clinical Oncology/College of American Pathologists clinical practice guideline update. J Clin Oncol 2013; 31: 3997-4013.

16. Goldhirsch A, Winer EP, Coates AS, et al. Personalizing the treatment of women with early breast cancer: highlights of the St Gallen International Expert Consensus on the Primary Therapy of Early Breast Cancer 2013. Ann Oncol 2013; 24: 2206-2223.

17. Kim SJ, Kim YS, Jang ED, et al. Prognostic Impact and Clinicopathological Correlation of CD133 and ALDH1 Expression in Invasive Breast Cancer. J Breast Cancer 2015; 18: 347.

18. Bednarz-Knoll N, Nastały P, Żaczek A, et al. Stromal expression of ALDH1 in human breast carcinomas indicates reduced tumor progression. Oncotarget 2015; 6: 26789-26803.

19. Badowska-Kozakiewicz AM, Patera J, Sobol M, Przybylski J. The role of oestrogen and progesterone receptors in breast cancer - immunohistochemical evaluation of oestrogen and progesterone receptor expression in invasive breast cancer in women. Contemp Oncol (Poznan) 2015; 19: 220-225.

20. Yoshioka T, Umekita Y, Ohi Y, et al. Aldehyde dehydrogenase 1 expression is a predictor of poor prognosis in node-positive breast cancers: a long-term follow-up study. Histopathology 2011; 58: 608-616.

21. Resetkova E, Reis-Filho JS, Jain RK, et al. Prognostic impact of ALDH1 in breast cancer: a story of stem cells and tumor microenvironment. Breast Cancer Res Treat 2010; 123: 97-108

22. Deng S, Yang X, Lassus H, et al. Distinct expression levels and patterns of stem cell marker, aldehyde dehydrogenase isoform 1 (ALDH1), in human epithelial cancers. PLoS One 2010; 5: e10277.

23. Charafe-Jauffret E, Ginestier C, Iovino F, et al. aldehyde dehydrogenase 1-positive cancer stem cells mediate metastasis 
and poor clinical outcome in inflammatory breast cancer. Clin Cancer Res 2010; 16: 45-55.

24. Lv X, Wang Y, Song Y, et al. Association between ALDH1+/ $\mathrm{CD} 133$ + stem-like cells and tumor angiogenesis in invasive ductal breast carcinoma. Oncol Lett 2016; 11: 1750-1756.

25. Park SY, Lee HE, Li H, et al. Heterogeneity for stem cell-related markers according to tumor subtype and histologic stage in breast cancer. Clin Cancer Res 2010; 16: 876-887.

26. Pan H, Wu N, Huang Y, et al. Aldehyde dehydrogenase 1 expression correlates with the invasion of breast cancer. Diagn Pathol 2015; 10: 66.

27. Ricardo S, Vieira AF, Gerhard R, et al. Breast cancer stem cell markers CD44, CD24 and ALDH1: Expression distribution within intrinsic molecular subtype. J Clin Pathol 2011; 64: 937-944.

28. Neumeister V, Agarwal S, Bordeaux J, et al. In situ identification of putative cancer stem cells by multiplexing ALDH1, $\mathrm{CD} 44$, and cytokeratin identifies breast cancer patients with poor prognosis. Am J Pathol 2010; 176: 2131-2138.

29. Mansour SF, Atwa MM. Clinicopathological significance of CD133 and ALDH1 cancer stem cell marker expression in invasive ductal breast carcinoma. Asian Pacific J Cancer Prev 2015; 16: 7491-7496.

30. Heerma van Voss MR, van der Groep P, Bart J, et al. Expression of the stem cell marker ALDH1 in BRCA1 related breast cancer. Cell Oncol (Dordr) 2011; 34: 3-10.

31. Honeth G, Lombardi S, Ginestier C, et al. Aldehyde dehydrogenase and estrogen receptor define a hierarchy of cellular differentiation in the normal human mammary epithelium. Breast Cancer Res 2014; 16: R52.

\section{Address for correspondence}

\section{Yazg1 Köy}

Department of Pathology

Batman State Hospital

Batman, Turkey

e-mail: dr.yazgikoy@gmail.com 